\title{
Real-time 3D Point Cloud Segmentation using Growing Neural Gas with Utility
}

\author{
Yuichiro Toda \\ Tokyo Metropolitan University \\ Graduate School of System Design \\ Tokyo, Japan \\ toda-yuuichirou@ed.tmu.ac.jp \\ Zhaojie Ju \\ University of Portsmouth \\ School of Computing \\ Portsmouth, UK
}

\author{
Hui $\mathrm{Yu}$ \\ University of Portsmouth \\ School of Creative Technologies \\ Portsmouth, UK
}

\author{
Naoyuki Takesue, Kazuyoshi Wada, Naoyuki Kubota \\ Tokyo Metropolitan University \\ Graduate School of System Design \\ Tokyo, Japan
}

\begin{abstract}
This paper proposes a real-time feature extraction and segmentation method for a 3D point cloud. First of all, we apply Growing Neural Gas with Utility (GNG-U) to the point cloud for learning a topological structure. However, the standard GNG-U cannot learn the topological structure of 3D space environment and color information simultaneously. To this end, we then modify the GNG-U algorithm by using a weight vector. we propose a surface feature extraction and segmentation method by efficiently utilizing the topological structure. Our segmentation method is based on a region growing method whose similarity value uses the inner value of two normal vectors connected by the topological structure. We show experimental results of the proposed method and discuss the effectiveness of the proposed method.
\end{abstract} words)

Keywords-component; formatting; style; styling; insert (key

\section{INTRODUCTION}

Recently, various types of robots has emerged in many fields as a progress of a robot technologies. Especially, the expectation of disaster robots, which can be robustly utilized in a disaster area, is increasing for preventing the second disaster in the area [1]. It is important to extract the environmental information related to a movable area of the robot and a dangerous area such as rubble with the high possibility of collapse in order to act safely and quickly in the disaster area. In this paper, we focus on an environmental sensing technology using a $3 \mathrm{D}$ point cloud for extracting the efficient and effective information in the disaster area. Specifically, we propose a real-time feature extraction and segmentation method using the time-sequatial $3 \mathrm{D}$ point cloud because segmenting the $3 \mathrm{D}$ point cloud enables the robot to utilize some objectives such as an environmental perception and recognition.

Many segmentation methods of the 3D point cloud have been proposed in recent years [2-4]. The researches of segmentation can be divided into two main streams from the viewpoint of the sensor devices. One stream is to combine the $3 \mathrm{D}$ distance sensor and a camera devise such as RGB-D sensor [5-7]. These researches can effectively cluster the material using color and luminance features and synthesizing the $3 \mathrm{D}$ coordinate system and camera coordinate system. However, it is unstable to cluster because these researches strongly depend on a lighting environment. Another stream is to use only a 3D distance sensor such as distance and surface feature based segmentation methods. For segmenting the 3D point cloud, [8-10] applied the region growing based algorithm using the distance and normal vector. These researches are very useful to divide the area in the 3D space because these kinds of methods do not depend on the lighting conditions. However, robust real time application is still not achieved [11] because almost methods do not consider the time series $3 \mathrm{D}$ point cloud.

In this paper, we propose a framework for 3D point cloud processing. Our proposed framework is based on Growing Neural Gas with Utility (GNG-U) because GNG-U can learn a topological structure of the $3 \mathrm{D}$ space environment and be applied to non-stationary data distribution. GNG-U proposed by Fritzke [12] is one of the competive learning methods, and can dynamically change the topological structure based on the edge referring to the ignition frequency of the adjacent node according to the accumulated error. Our proposed method also uses the surface features for segmenting the 3D point cloud. However, the standard GNG-U do not learn the topological structure of the 3D space environment and color information 
simaltaneously. Therefore, we also propose the modified GNG-U, which uses the weight vector for selecting the first and second winner nodes and the rule of node deletion is modified for adjusting the time series 3D point cloud quickly. Next, we explain the surface feature extraction method. Our feature extraction method is based on a local surface method proposed by [13]. In our method, we utilize the topological structure of GNG-U, and the local surface is composed of the nearest ndoes connected to a selected node. Furtheremore, we propose the segmentation method of $3 \mathrm{D}$ point cloud. Our proposed method is also based on the region growing method. A similarity value of the growing region method uses an inner value between two normal vectors which have connection of the edge. Furtheremore, the region is growed by tracking the connectivities of the topological strucuture. In this way, we utilize the toplolgical structure of the modified GNG-U efficiently for realizing the real-time feature extraction and segmentation method of 3D point cloud. Finally, we show experimental results of the segmentation of $3 \mathrm{D}$ point cloud using the proposed methods, and discusses the effectiveness of the proposed methods.

\section{SYSTEM OVERVIEW}

This section outlines our proposed method. Fig.1 shows a total flowchart of our proposed method. In this paper, we use Kinect developed by Microsoft as a RGB-D sensor [14]. Table 1 shows the specification of Kinect. This sensor captures the depth information and color image simultaneously, and can integrates the depth and color coordination information eaisly. After measuring the RGB-data, we downsample the point cloud by using a voxel grid for reducing the total processing cost. Our segmentation can be divided into two main approaches. One is the distance based segmentation method using GNG-U for learning a topological structure. The topological structure is utilized for extracting a surface feature and segmenting the point cloud. Another is a normal vector based segmentation method. This segmentation method is based on a region growing method utilizing the topological structure and a similarity value of the region growing method uses the normal vector for segmenting the point cloud.

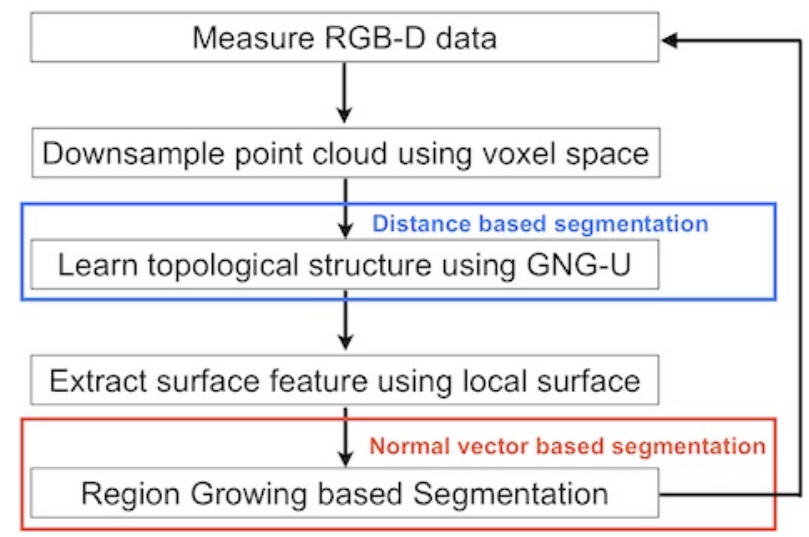

Fig. 1. Total flowchart of our proposed method.
TABLE I. SPECIFICATION OF KINECT

\begin{tabular}{|l|l|}
\hline Size & $282 \times 72 \times 72[\mathrm{~mm}]$ \\
\hline Measurement range & $57 \times 43[\mathrm{deg}]$ \\
\hline Measurement distance & $0.5-7.0[\mathrm{~m}]$ \\
\hline Resolution & $320 \times 240,640 \times 480[\mathrm{pixel}]$ \\
\hline Sampling rate & $30[\mathrm{fps}]$ \\
\hline
\end{tabular}

\section{Modified Growing Neural Gas with Utility}

We use a Growing Neural Gas(GNG) based algorithm for learning a topological structure of the $3 \mathrm{D}$ point clouds. GNG proposed by Fritzke is one of the unsupervised learning methods [15]. Unsupervised learning is performed by using only data without any teaching signals. Self-organized map (SOM), neural gas (NG), growing cell structures (GCS), and GNG are well known as unsupervised learning methods [1618]. Basically, these methods use the competitive learning. The number of nodes and the topological structure of the network in SOM are designed beforehand. In NG, the number of nodes is fixed beforehand, but the topological structure is updated according to the distribution of sample data. On the other hand, GCS and GNG can dynamically change the topological structure based on the adjacent relation (edge) referring to the ignition frequency of the adjacent node according to the error index. However, GCS does not delete nodes and edges, while GNG can delete nodes and edges based on the concept of ages. Furthermore, GCS must consist of $\mathrm{k}$-dimensional simplexes whereby $k$ is a positive integer chosen in advance. The initial configuration of each network is a $k$-dimensional simplex, e.g., a line is used for $k=1$, a triangle for $k=2$, and a tetrahedron for $k=3$. GCS has applied to construct 3D surface models by triangulation based on 2dimensional simplex. However, because the GCS does not delete nodes and edges, the number of nodes and edges is over increasing. Furthermore, GCS cannot divide the sample data into several segments. In addition, GNG cannot apply the nonstationary data distribution because GNG can only remove the nodes that are the nearest node of the first winner node in GNG. Therefore, we use GNG with Utility (GNG-U) for learning the topological structure from 3D space environment.

The procedure and notation used in GNG-U are shown in Algorithm 1, where $h_{i}$ indicates the nth dimensional vector of a node; $w$ indicates the $n$th dimensional weight vector; $A$ indicates a set of nodes; $c_{i j}$ indicates a set of edges between the ith and jth nodes; $g_{i j}$ indicates Age of the edge between the ith and jth nodes. GNG based algorithm select the nearest unit (winner) $s_{1}$ and the second-nearest unit $s_{2}$ from the set of nodes and create the connection $\left(c_{s 1, s 2}=1\right)$ if a connection between $s_{1}$ and $s_{2}$ does not yet exist. In GNG-U, each node has the utility value $U_{i}$. The node with the minimum utility value is removed from the topological structure if the following condition is satisfied;

$$
E_{u} / U_{l}>k
$$

Generally, the standard GNG-U removes the node in the node insertion. In addition, our method removes the node if the number of input data generated so far is an integer multiple of a parameter $\kappa$ for controlling the number of nodes. Furthermore, our method uses the weight vector $w$ to learn the 
topological structure of the 3D space environment. Fig.2 and 3 show a 3D point cloud data with color and an example of GNG-U with and without the weight vector. GNG-U with the weight vector can learn the topological structure of the 3D environmental space with color (Fig.3 (a)), while GNG-U without the weight vector cannot learn the topological structure of the 3D environmental space.
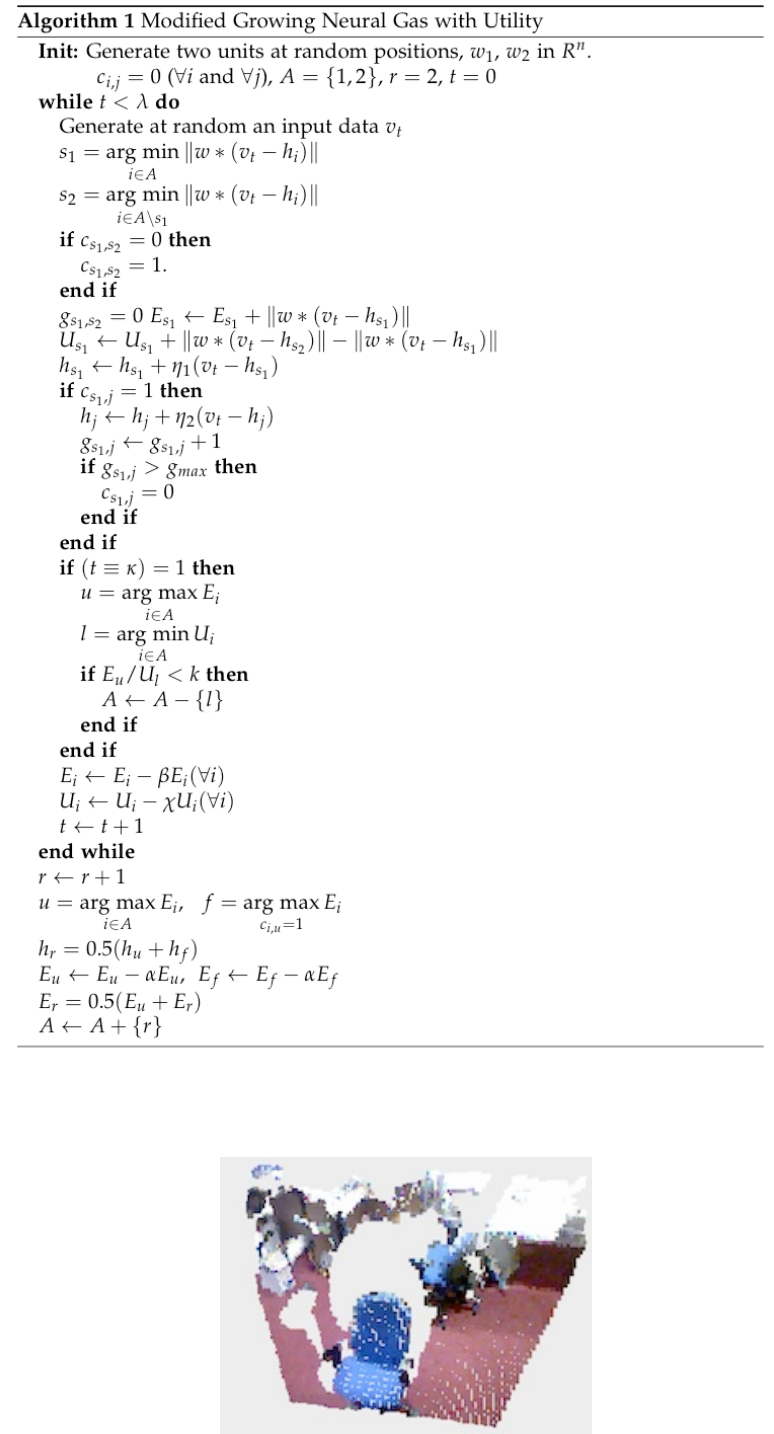

Fig. 2. An example of the point cloud with color.

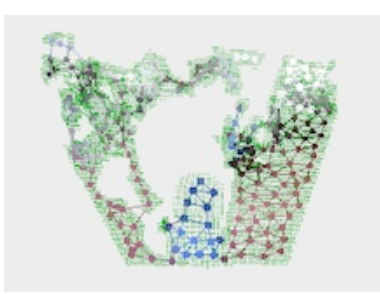

(a) With weighted vector

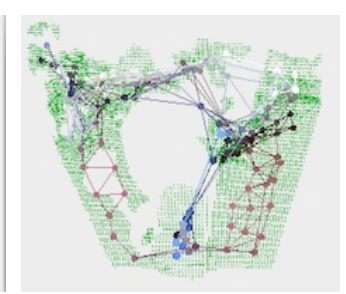

(b) Without weighted vector
Fig. 3. An exmaple of GNG-U with/without weighted vector. Green dot indicates 3D point cloud. Other nodes and edge indicate the topologica structure of GNG-U.

\section{SEGMENTATION METHOD USING A TOPOLOGICAL STRUCTURE}

\section{A. Feature extraction}

After learning the topological structure, the normal vectors and curvatures are extracted as a feature of the $3 \mathrm{D}$ point cloud. At first, a local surface of the node $i$ is composed of the nearrest nodes $\left(c_{i, j}=1\right)$. Fig. 4 shows the concept image of the local surface. Next, the weighterd center of gravity $o_{i}$ of the local surface is calculated, and then covariance matrix is calculated as follows.

$$
\begin{aligned}
& C_{i}=\sum_{j=1}^{k}\left(h_{j}-o_{i}\right)^{\mathrm{T}}\left(h_{j}-o_{i}\right) \\
& o_{i}=\frac{h_{i}+\sum_{j=1}^{k} w_{i, j} \cdot h_{j}}{1+\sum_{j=1}^{k} w_{i, j}}, w_{i, j}=\exp \left(-\frac{\left\|\mathbf{x}_{i}-\mathbf{x}_{j}\right\|^{2}}{\mu^{2}}\right)
\end{aligned}
$$

where $\mu$ indicates the coefficient. After calculating the covariance matrix, the eigen vectors and values of the matrix are calculated for estimating the normal vector $\left(\mathbf{C}_{i} \mathbf{v}=\lambda \mathbf{v}, \lambda_{1} \leq \lambda_{2}\right.$ $\left.\leq \lambda_{3}\right)$. And then the normal vector is assined to the eigen vector with the minimu eigen value [13]. Furtheremore, the curvature of the surface is calculated by the following eqation:

$$
r_{i}=\frac{\lambda_{1}}{\lambda_{1}+\lambda_{2}+\lambda_{3}}
$$

In this way, the features of $3 \mathrm{D}$ point cloud are calculated. Our segmentation method uses the normal vector.

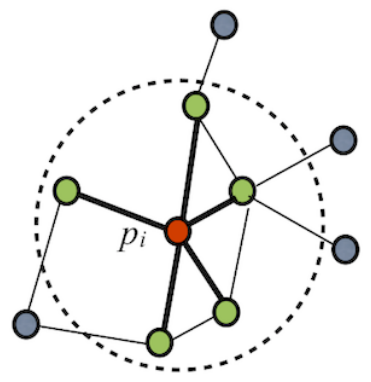

Fig. 4. Concept image of the local surface. Red and green nodes indicate the selected node and the nearest node of the selected node, respectively.

\section{B. Region Growing based segmentation method}

After estimating the normal vector, we segment the 3D point cloud utilizing the topological structure of 3D space environment. Our proposed method is basically based on the region growing approach. In the method, the similaity $s_{i, j}$ between the $i$ th and $j$ th nodes is defined as the inner value of the normal vectors between nodes.

$$
s_{i, j}=\mathbf{n}_{i} \cdot \mathbf{n}_{j}
$$

This similarity means the measure of the angle between the normal vectors. The total algorithm is as follows.

Step0. $l_{i}$ of the node set to $-1\left(l_{i}=-1, \forall i \in A\right)$, and label number $n$ set to $0(n=0)$. The temporary set to $0(\Pi=\phi)$. 
Step1. Select the $i$ th node whose label is -1 from the set $A$ randomly, and the $j$ th label sets to $n\left(l_{i}=n\right)$.

Step2. Select the all nearest nodes from the $i$ th node $\left(c_{i, j}=1\right)$, and if the similarity value between the $i$ th and $j$ th nodes is less than threshold value $\sigma$, the $j$ th label sets to $n\left(l_{j}=n\right)$. In addition, the $j$ th node set to the temporary set $(\Pi \leftarrow \Pi-\{j\})$;

Step 3. Select and remove the $i$ th node from the set $\Pi$ ( $\leftarrow \leftarrow \Pi-\{i\})$ and go to step 2 if the temporary set is not empty. Otherwise, go to step 4.

Step 4. Increment the label $n$ and go to step 1 if unlabeled node $\left(l_{i}=-1\right)$ exists. Otherwise, stop the segmentation.

In this paper, the threshold value $\sigma$ is determined empirically and fixed throughout the experiment in this paper. In this way, our proposed method combines the distance based clustering method (GNG-U) and the region growing based segmentation method using the normal vector of the local surface.

\section{EXPERIMENTAL RESULTS}

This section shows experimental results of our proposed method. All exiperimtents were conducted on a computer with 2.3 GHz Intel Core i7 and 8GB RAM. The parameters setting up in the experiment includes $\lambda=300, \kappa=10, \eta_{1}=0.08, \eta_{2}=$ $0.008, g_{\max }=88, \alpha=0.5, \beta=0.005, \chi=0.005, \mu=0.5, k=$ 1000. As mentioned above, these parameters were determined empirically. In this experiment, the $3 \mathrm{D}$ point cloud was collected by moving Kinect with hand. Fig.5 shows the examples of the topological structures using our proposed method, the standard GNG-U and GNG. In these results, GNG cannot be applied to the non-stationary data distribution because GNG generates the redundant nodes and edges (Fig. 4 (a)). The result of the standard and our GNG-U can apply to the non-stationary data distiribution because the nodes are removed from the topological structure according to the utility value. In addition, Fig. 6 shows the time series results of the standard GNG-U and our proposed method. In Fig.6 (b), both methods cannot learn the topological structure efficiently because the data distribution greatly changes (from Fig.6 (a) to Fig.6 (b)). After a few frames, the topological structure of the standard GNG-U still includes some redundant nodes and edges. The modified GNG-U relearns the topological structure correctly because the modified GNG-U has a high frequency of removing the nodes according to the parameter $\kappa$. Fig.7 demonstrates the number of nodes and data in each step. This result also indicates that the modified GNG-U can control the number of nodes according to the non-stationary data distribution. In this way, our proposed method can learn the topological structure of the 3D space environment from the non-stationary data distribution efficiently.

We have also done some experiments on our region growing based segmentation method. Fig. 8 shows an example of feature extraction and segmentation result by proposed method. As can be seen from this figure, our proposed method can estimate the normal vector and roughly segment the $3 \mathrm{D}$ point cloud such as walls and floor. In addition, Fig.9 shows the transition of the processing time which includes all the processings. The average time is 0.022 [sec] and the maximum time is 0.031 [sec]. We have achieved real-time processing for the $3 \mathrm{D}$ point cloud by utilizing the topological structure efficiently because the frame rate of Kinect is about 0.33 [sec].

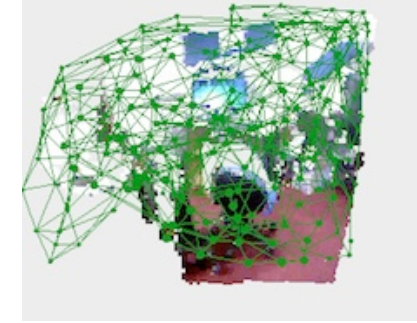

(a) GNG

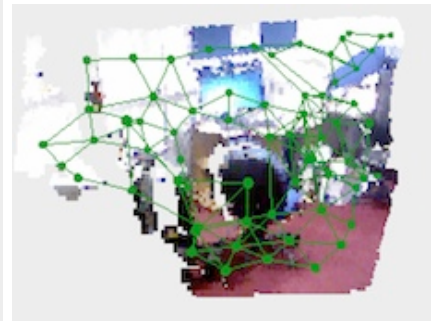

(b) Standard GNG-U

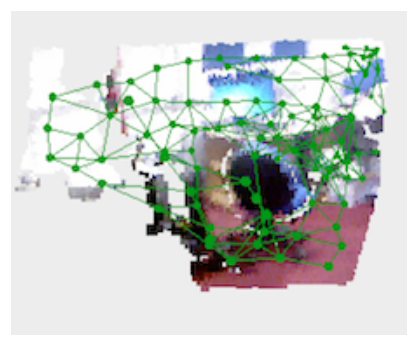

(c) Modified GNG-U

Fig. 5. An example of the topological structure in each method.

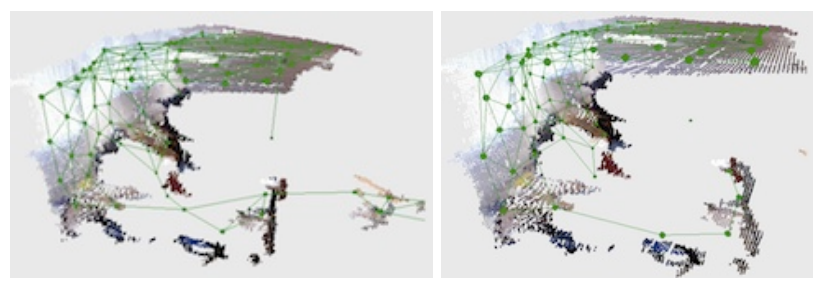

(a) Step 146 (Left: Standard GNG-U, Right: Modified GNG-U)

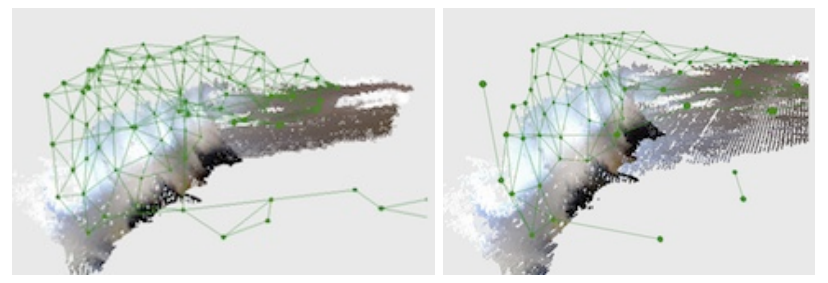

(b) Step 148 (Left: Standard GNG-U, Right: Modified GNG-U)

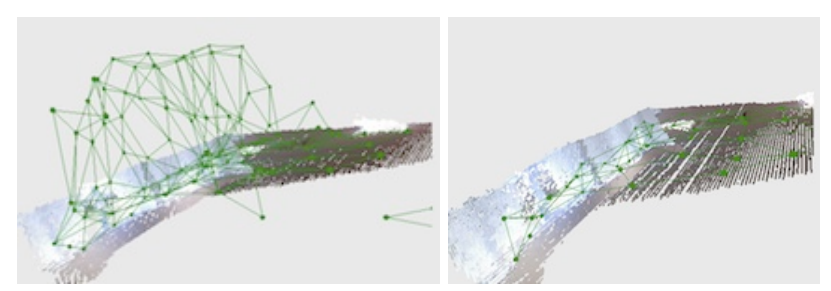

(c) Step 150 (Left: Standard GNG-U, Right: Modified GNG-U)

Fig. 6. Examples of the topological stucure for the time series data. 


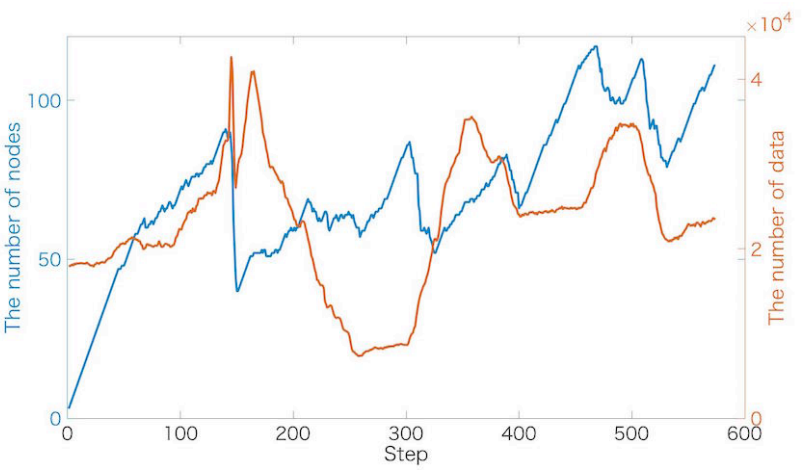

Fig. 7. The transition of the numbers of data and nodes.

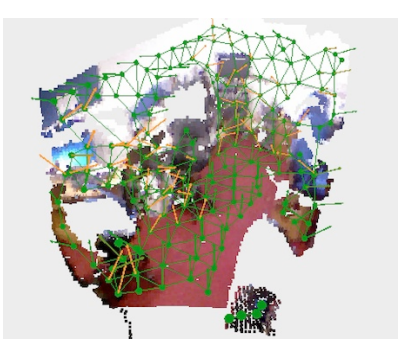

(a) Feature extraction

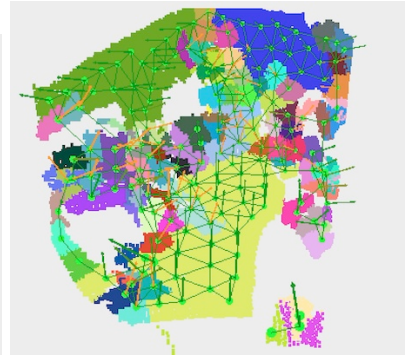

(b) Segmentation result
Fig. 8. An example of featuer extraction and segementation result.

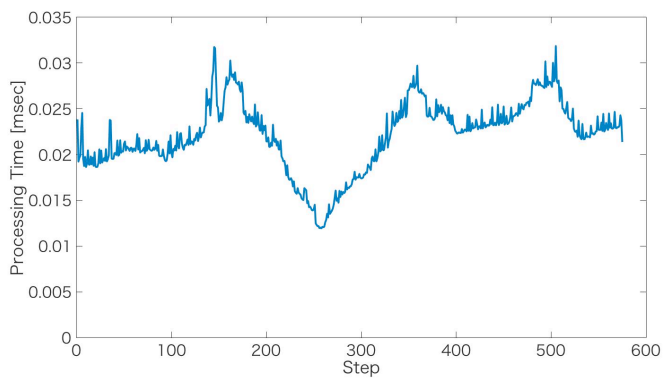

Fig. 9. The transition of the processing time.

\section{CONCLUSION}

In this paper, we proposed the real-time feature extraction and segmentation method from the 3D point cloud. We first proposed the modified GNG-U algorithm for learning the topological structure with color information and applying to the non-stationary data distribution efficiently. The we proposed the surface feature extraction and the region growing based segmentation method by utilizing the topological sturucture efficiently. As can be seen from the experimental result, the modified GNG-U ourperformed GNG and the standard GNG-U for the time serise 3D point cloud. However, our segmentation method could provide rough segmentation. Therefore, we will combine the rough and detail segmentation method by proposing the hierarchical approach of the modified GNG-U algorithm as the future work.

\section{Acknowledgment}

This work was funded by ImPACT Program of the Council for Science, Technology and Innovation.

This work was supported by EU seventh framework programme under grant agreement No. 611391, Development of Robot-Enhanced Therapy for Children with Autism Spectrum Disorders (DREAM);

\section{References}

[1] Murphy, Robin R., et al. "Search and rescue robotics." Springer Handbook of Robotics. Springer Berlin Heidelberg, 2008. 1151-1173.

[2] Ning, Xiaojuan, et al. "Segmentation of architecture shape information from 3D point cloud." Proceedings of the 8th International Conference on Virtual Reality Continuum and its Applications in Industry. ACM, 2009.

[3] Dorninger, P., and C. Nothegger. "3D segmentation of unstructured point clouds for building modelling." International Archives of the Photogrammetry, Remote Sensing and Spatial Information Sciences 35.3/W49A (2007): 191-196.

[4] Nguyen, Anh, and Bac Le. "Contextual Labeling 3D Point Clouds with Conditional Random Fields." Intelligent Information and Database Systems. Springer International Publishing, 2014. 581-590.

[5] J. Strom, A. Richardson, E. Olson, Graph based segmentation ofcolored 3d laser point clouds, In Proc. of the IEEE/RSJ International Conference on Intelligent Robots and Systems (IROS), 2010.

[6] To, Andrew Wing Keung, Gay Paul, and Dikai Liu. "Surface-type classification using RGB-D." Automation Science and Engineering, IEEE Transactions on 11.2 (2014): 359-366.

[7] Diebold, Julia, et al. "Interactive multi-label segmentation of RGB-D images." Scale Space and Variational Methods in Computer Vision. Springer International Publishing, 2015. 294-306.

[8] Tóvári, D., and N. Pfeifer. "Segmentation based robust interpolation-a new approach to laser data filtering." International Archives of Photogrammetry, Remote Sensing and Spatial Information Sciences 36.3/19 (2005): 79-84.

[9] Koppula, Hema S., et al. "Semantic labeling of 3d point clouds for indoor scenes." Advances in neural information processing systems. 2011.

[10] Rusu, Radu Bogdan, et al. "Towards 3D point cloud based object maps for household environments." Robotics and Autonomous Systems 56.11 (2008): 927-941.

[11] Nguyen, Anh, and Bac Le. "3D point cloud segmentation: A survey." Robotics, Automation and Mechatronics (RAM), 2013 6th IEEE Conference on. IEEE, 2013.

[12] Fritzke, Bernd. "A self-organizing network that can follow nonstationary distributions." Artificial Neural Networks-ICANN'97. Springer Berlin Heidelberg, 1997. 613-618.

[13] Pauly, Mark, Markus Gross, and Leif P. Kobbelt. "Efficient simplification of point-sampled surfaces." Proceedings of the conference on Visualization'02. IEEE Computer Society, 2002.

[14] Microsoft Kinect for Windows, https://developer.microsoft.com/enus/windows/kinect

[15] B. Fritzke: A growing neural gas network learns topologies; Advances in Neural Information Processing Systems, Vol. 7, pp. 625-632, 1995.

[16] T. Kohonen: Self-Organizing Maps; Springer, 2000.

[17] T. M. Martinetz and K. J. Schulten: A "neural-gas" network learns topologies; Artificial Neural Networks, Vol. 1, pp. 397-402, 1991.

[18] B. Fritzke: Unsupervised clustering with growing cell structures; Neural Networks, Vol. 2, pp 531-536, 1991 